\title{
A first study on the use of TerraSAR-X for meteorological purposes
}

\author{
José Márquez-Martínez \\ Microwaves and Radar Institute \\ German Aerospace Center (DLR) \\ Oberpfaffenhofen, Germany \\ Email: jose.marquez@dlr.de
}

\author{
José Luis Álvarez-Pérez \\ Deparment of Signal Theory and Communications \\ Technical University of Catalonia (UPC) \\ Barcelona, Spain \\ Email: jlalvarez@tsc.upc.edu
}

\begin{abstract}
In this paper we present the predicted performance of the TerraSAR-X for meteorological purposes. Principally, the study focuses on the possibility of measuring rain over ocean and forest. Regarding the acquisition geometry, we investigate the rain volume and voxel resolution, the signal-to-noise ratio and the signal-to-clutter ratio. The clutter interference has been evaluated for intra- and inter-pulse ground returns. Measurement of rain for nadir-looking geometry looks promising over forest and ocean under almost all conditions. However, for side-looking geometry, rain detection could be only possible at very high rain rates.
\end{abstract}

\section{INTRODUCTION}

There is a need for precipitation measurements over many parts of the world, especially over oceans and remote areas [1]. Observations of rainfall are crucial in order to provide a baseline for studies on climate, meteorology and the hydrological cycle. So far different remote sensing techniques have been used for this purpose, namely visible and infrared sensors as well as microwave radiometers and ground-based radars. However, while ground-based radars have a limited coverage, space-borne radars should allow the global monitoring of rainfall rate. The advantage of radar with respect to the other techniques lies in the fact that the radar echo is a direct measure of the rainfall intensity. Additionally, the timing discrimination ability of radar provides information about the vertical profile. Nevertheless, in contrast to what is often believed, microwave radiation may be affected by the presence of the clouds themselves. The only space-borne precipitation radar to date has been flown on board the Tropical Rain Measuring Mission (TRMM) satellite [2]. However, no synthetic aperture radar (SAR) has been used systematically for precipitation monitoring yet. SAR systems could improve spatial resolution significantly and provide estimates on water drop velocities.

Although several space-borne SAR instruments have been operated for ocean and land surface imaging, the chosen frequency bands and the lack of scanning capabilities have precluded their application for rainfall field measurements. In October 2006 it is planned to launch the German TerraSAR$\mathrm{X}$ satellite, a public private partnership between the German Aerospace Center (DLR) and ASTRIUM GmbH. It is equipped with a highly flexible phased array antenna which allows for SAR Stripmap, ScanSAR and Spotlight operations.
Due to the flexibility of the system, a number of scientific proposals have been put forward. Among them, a meteorological experiment excels as one of the most interesting attempts to continue the line of research reported after the SIR-C/X-SAR mission [3].

\section{The RAIN Volume}

The rain volume has a main role in the precipitation SAR (PSAR) performance and can be expressed as follows

$$
V_{\mathrm{i}}=S \cdot \Delta R,
$$

where $\Delta R$ is the slant range resolution and $S=\Omega \cdot R^{2}$ the effective surface seen by the radar, which is related to the solid angle

$$
\Omega=\int_{\varphi} \int_{\xi} \cos \xi \mathrm{d} \xi \mathrm{d} \varphi
$$

and the slant range distance $R$. The rain volume depends on the geometry of the acquisition, i.e. on the look angle, see Figure 1. Similar to [4], the rain volume may be divided into

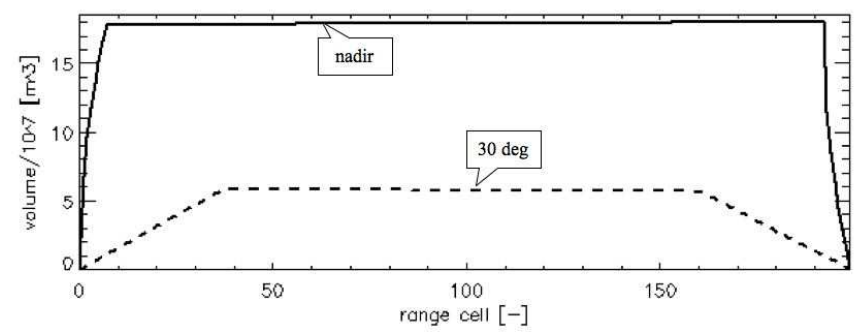

Fig. 1. Rain volume for nadir- and side-looking geometry

three regions within the $3 \mathrm{~dB}$ beamwidth:

1) Near range: the rain volume is limited by the cloud top;

2) Mid range: the rain volume is maximum and the largest for the given geometry;

3) Far range: the rain volume is limited by the farthest edge of the beam.

Note that for the volume in zone 2), the nadir-looking case is maximum when a complete beam filling is obtained, i.e the solid angle is limited by the $3 \mathrm{~dB}$ antenna beamwidth. On 
the other hand, the side-looking case is limited by the ground and the rain top which is typically smaller than the elevation beamwidth. Additionally, the zones 1) and 3) are in case of side-looking larger so the rain volume for the nadir geometry is always bigger than the side-looking ones. Therefore, the rain volume varies with the look angle as shown in Figure 2.

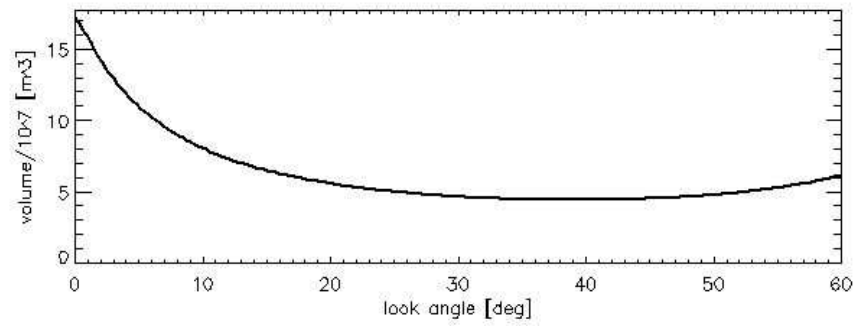

Fig. 2. Rain volume vs. look angle

\section{SAR RESOLUTION IN RAIN}

The rain volume consists of a conglomerate of small size rain cells which different rainfall rates. The PSAR shall provide enough resolution to facilitate the estimation of rainfall and drop size distribution. Since rain is a volumetric target, the resolution may be divided into three components which define the voxel size

$$
\delta V=\delta x \cdot \delta y \cdot \Delta R,
$$

where $\delta x$ is the along-track resolution and $\delta y$ the crosstrack one. If the voxel size is too big, a lot of different rain cells were averaged and thus, we would lose some important information to understand the rain rate distribution and dynamic of the storm. The objective is to have the smallest voxel by minimising the 3-D components.

\section{A. Along-track resolution}

The along-track resolution $\delta x=\beta_{s} R$ depends on the maximum possible aperture that can by synthesised during the target illumination. Since raindrops are falling, they are changing their position with time. The time needed to effective change their position is called decorrelation time. Coherent integration can only be maintained for the decorrelation time. The shorter the decorrelation time, the larger the resolution element. Atlas and Moore [5], showed that the synthetic beamwidth can be expressed as $\beta_{s}=2 \sigma_{v} / v$, where $\sigma_{v}$ is the standard deviation of the precipitation velocities, and $v$ is the platform velocity. They also showed that an effective $\beta_{s}$ better than the real aperture is only achieved when standard deviation of the Doppler velocity spectrum is less than

$$
\sigma_{v} \lesssim \frac{\lambda \cdot v}{6 \cdot l},
$$

where $\lambda$ is the wavelength, and $l$ is the length of the antenna in along-track. Therefore, for the TerraSAR- $X$ case, the resolution can effectively be improved when $\sigma_{v} \lesssim 7.5 \mathrm{~m} / \mathrm{s}$, i.e. for small to moderate storms. The Doppler velocity spectrum
TABLE I

TERRASAR-X SYSTEM PARAMETERS

\begin{tabular}{|lcc|}
\hline Parameter & Symbol & Value \\
\hline Wavelength & $\lambda$ & $3.1 \mathrm{~cm}$ \\
Peak transmission power & $P_{t}$ & $2.26 \mathrm{~kW}$ \\
Pulse width & $\tau$ & $51.4 \mu \mathrm{s}$ \\
Pulse repetition frequency & $\mathrm{PRF}$ & $3500 \mathrm{~Hz}$ \\
Antenna gain & $G$ & $45 \mathrm{~dB}$ \\
Antenna height & $l_{h}$ & $0.7 \mathrm{~m}$ \\
Antenna length & $l_{v}$ & $4.7 \mathrm{~m}$ \\
Receiver bandwidth & $B$ & $150 \mathrm{MHz}$ \\
Noise figure & $F$ & $5.3 \mathrm{~dB}$ \\
Satellite height & $h$ & $514 \mathrm{~km}$ \\
Satellite velocity & $v$ & $7.3 \mathrm{~km} / \mathrm{s}$ \\
\hline
\end{tabular}

varies with the nature of the precipitation and the associated windfield and turbulence and thus, the along-track resolution will be variable and a priori unknown.

\section{B. Across-track resolution}

The across-track resolution $\delta y=\beta_{v} R$ depends on the across-track antenna beamwidth $\beta_{v}$ as well as the geometry of the acquisition. The effective $\beta_{v}$ varies through the range cell. Concerning the zone 2), which is the most important one in terms of performance, it ranges from total beam-filling in the nadir-looking case up to ground to rain top limit for the side-looking geometry.

\section{Slant range resolution}

The slant range resolution $\Delta R=c /(2 B)$ is determined by the transmitted chirp bandwidth $B$ which in TerraSAR-X can be as large as $300 \mathrm{MHz}$. This results in resolutions of up to 0.5 meters which may be used, for instance, to map very accurately the melting layer in nadir-looking geometry. Multilooking processing techniques may also be utilised in order to improve the radiometric resolution of the measurements by incoherent addition of the different parts of the echoes spectrum.

\section{Models Used}

The TerraSAR-X parameters utilised for the following performance predictions are given in Table I. All simulations were done at Equator and taking a spherical Earth model. The effective cloud altitude was set to $4 \mathrm{~km}$ and the sigma nought over forest to $\sigma^{0}=-10 / \cos \beta \mathrm{dB}$.

\section{A. Rain parameters}

To calculate rain returns we used the empirical power law

$$
z=A \cdot R_{r}^{b},
$$

where $R_{r}$ is the rainfall rate $(\mathrm{mm} / \mathrm{h}), \mathrm{z}$ is the radar reflectivity factor $\left(\mathrm{mm}^{6} / \mathrm{m}^{3}\right)$, and A and $\mathrm{b}$ are empirical constants which depend on the frequency and the dropsize distribution, i.e. on the rain type [6]. We chose $A=300$ and $b=1.5$ like in [4] and [5] for all calculations. 
The rain attenuation follows the Mueller-Jones' model for $\mathrm{X}$-Band at $0^{\circ} \mathrm{C}$ as presented in [6]. This reads

$$
k_{r}=2 \cdot 0.0058 \cdot L_{r} \cdot R_{r}
$$

where $k_{r}$ is the two way rain attenuation in $\mathrm{dB}$, and $L_{r}$ is the rain path in $\mathrm{km}$.

The melting layer or bright band attenuation [7] depends on the rainfall rate. Bellon et al. [8] stated that the total bright band attenuation is of the order of 10 times the specific attenuation associated with the rainfall rate below the bright band. The results presented there were obtained for vertical incidence so stronger attenuation is expected as the look angle increases. The next exponential model obtained by curvefitting the results presented in [8] is used for the melting layer attenuation

$$
k_{m}=\frac{0.07 \cdot 1.09^{Z}}{\cos (\beta)},
$$

where $k_{m}$ is the bright band two way attenuation, and $Z$ is the radar reflectivity factor in $\mathrm{dB}$. A simple $1 / \cos (\beta)$ correction factor is introduce in order to account for the look angle $\beta$. Note that the two way attenuation for a rainfall rate of 100 $\mathrm{mm} / \mathrm{h}$ corresponds to approximately $8 \mathrm{~dB}$ of attenuation in nadir-looking geometry.

\section{B. Ocean scattering model}

To simulate the backscattering coefficient of the ocean a two-scale model has been implemented [10]. This model combines gravity waves of the sea surface via the Kirchhoff approximation as well as capillary waves through the firstorder small perturbation model. Two wind speeds have been considered, namely 12 and $24 \mathrm{~m} / \mathrm{s}$. The power spectrum of the ocean surface has being taken to match the shape worked out by Pierson-Moskowitz [11], so the wind-speed refers to a height of $19.5 \mathrm{~m}$, at which their anemometers were mounted on the weather ships.

\section{Signal-TO-Noise Ratio}

The precipitation radar equation for signal-to-noise ratio (SNR) can be expressed as follows

$$
\mathrm{SNR}=\frac{P_{t}}{k T B F} \cdot \frac{\lambda^{2} G^{2} \sigma^{0}}{(4 \pi)^{3} R^{4}} \cdot V_{i} \cdot L_{r},
$$

where $L_{r}=10^{-0.1 \cdot\left(k_{r}+k_{m}\right)}$ is the rain loss due to the melting layer and the rain attenuation, $k$ is the Boltzmann's constant, and $T=290 \mathrm{~K}$ is the receiver temperature. The scattering coefficient $\sigma^{0}$ is

$$
\sigma^{0}=\frac{\pi^{5} \cdot 300 \cdot R_{r}^{1.5} \cdot|k|^{2} \cdot 10^{-18}}{\lambda^{4}}
$$

where $|k|^{2}=0.93$ for water [6]. Figure 3 shows the SNR for five different look angles. As it is shown, the SNR is only adequate for small look angles and principally only for nadir-looking geometry. For those look angles beyond $15^{\circ}$ and for rainfall rates greater than $20 \mathrm{~mm} / \mathrm{h}$, the SNR is severely degraded by the rain and the melting layer attenuation. The bright band pick reflectivity is proportional to the rainfall rate

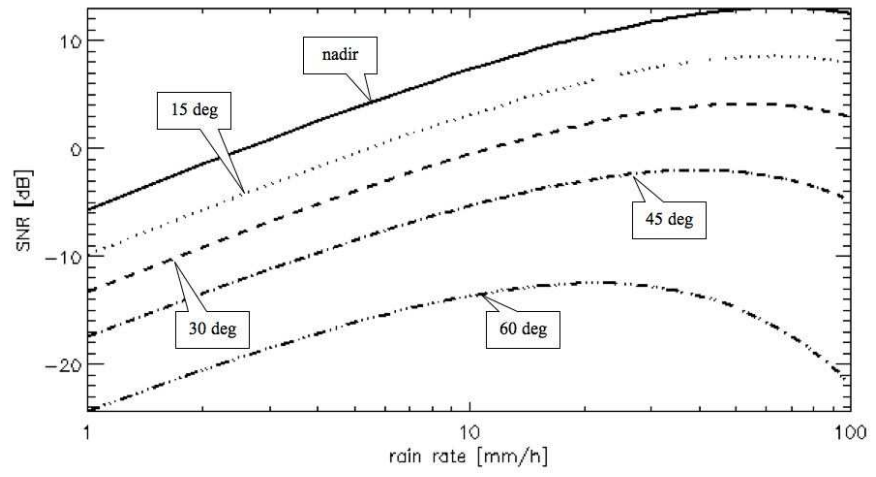

Fig. 3. SNR vs. rain rate for five different look angles

and can be of the order of $10 \mathrm{~dB}$ stronger than the reflectivity of the rain bellow [7]. Therefore, in case of severe attenuation due to melting layer, the measurement of the bright band pick could be used as an indirect measurement of the associated rain of the storm. Since the slant range resolution can be as short as $0.5 \mathrm{~m}$, we could also focus on the study of the bright band form and composition.

\section{Vi. Signal-to-Clutter Ratio}

The signal-to-clutter (SCR) ratio measures the surface return components with respect to the desired rain signal. For this purpose, the SCR has been divided into two clutter component. The first one corresponds to the classical clutter ratio, i.e. intrapulse interference, while the second one corresponds to the signal-to-range ambiguity ratio, i.e. interpulse interference.

\section{A. Intrapulse interference}

The intrapulse interference can be computed as the ratio of the desired signal to the surface return within the same echo. Therefore, the SCR for intrapulse interference can be expressed as

$$
\mathrm{SCR}_{\mathrm{r}}=\frac{\sigma^{0} \cdot V_{i}}{\sigma_{g}^{0} \cdot A_{g}},
$$

where $\sigma_{g}^{0}$ is scattering coefficient of the surface, and $A_{g}$ is the ground surface. Figure 4 shows the percentage of clutter within the desired rain echo. The nadir-looking geometry is not suffering from this interference. The percentage of clutter content within the desired data increases with the look angle.

\section{B. Interpulse interference}

The interpulse interference or signal-to-range ambiguity ratio [9] can be computed as follows

$$
\mathrm{SCR}_{\mathrm{t}}=\frac{\sigma^{0} G_{r}^{2} V_{i} L_{r}^{r}}{R_{r}^{4}} \cdot \frac{R_{g}^{4}}{\sigma_{g}^{0} G_{g}^{2} A_{g} L_{r}^{g}},
$$

where the sub- and super-index $r$ reads for rain and $g$ for ground clutter. The $\mathrm{SCR}_{\mathrm{t}}$ is the major contributor to the $\mathrm{SCR}$ for the nadir-looking geometry. 


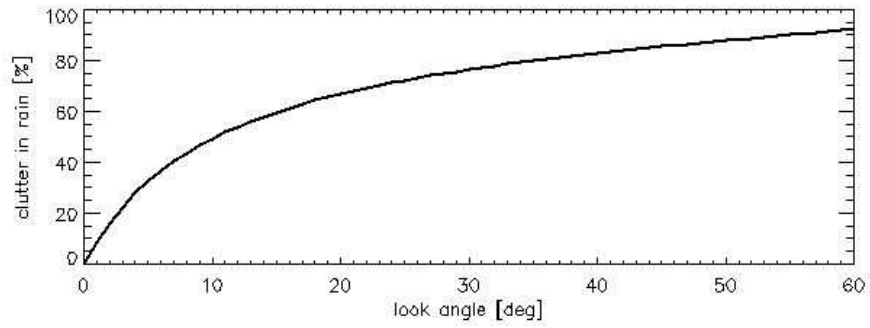

Fig. 4. Percentage of clutter within the rain echo

\section{Total signal-to-clutter ratio}

The total signal-to-clutter ratio has been computed as the worst case combination of the aforementioned contributions.

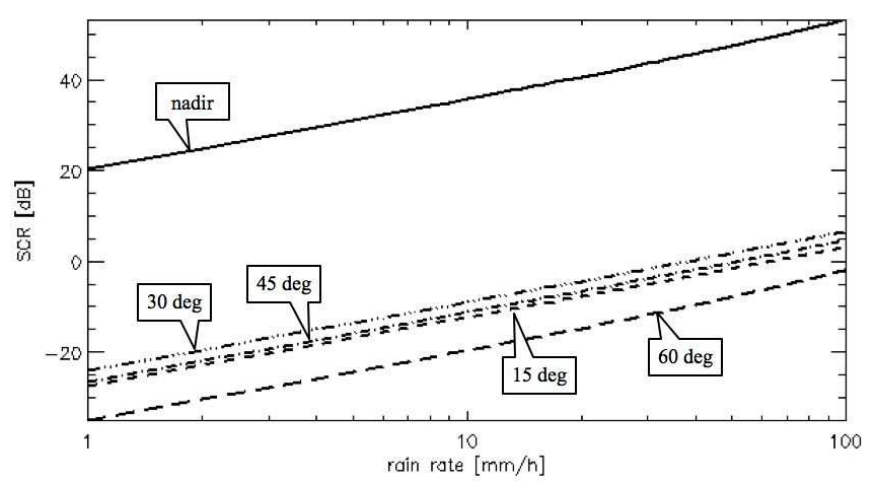

Fig. 5. SCR vs. rain rate over forest

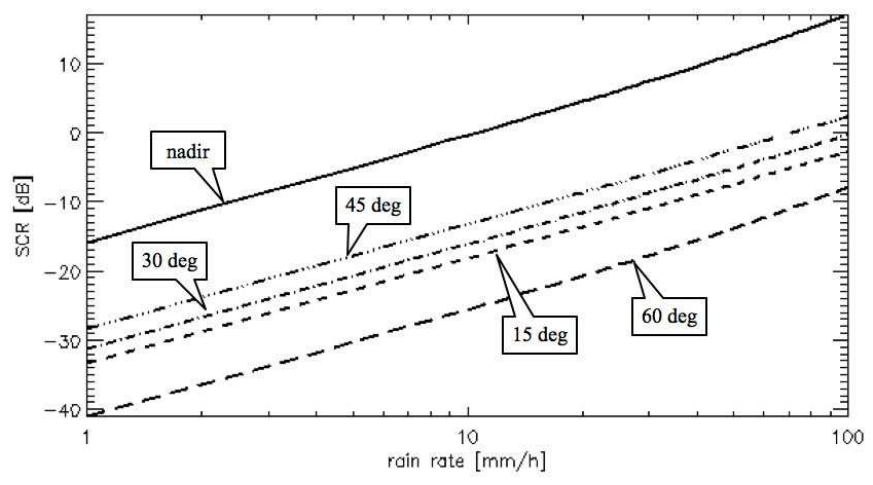

Fig. 6. SCR vs. rain rate over ocean for $12 \mathrm{~m} / \mathrm{s}$ wind speed

Figure 5 shows SCR over a forest. The rain detection over forest is feasible only for close to nadir-looking geometry. Figures 6-7 show plots of the SCR over ocean for wind velocities of 12 and $24 \mathrm{~m} / \mathrm{s}$. For the $12 \mathrm{~m} / \mathrm{s}$ case, only the nadir case would perform sufficiently good to measure rain, but only for rainfall rates greater than $10 \mathrm{~mm} / \mathrm{h}$. The performance is even better for the $24 \mathrm{~m} / \mathrm{s}$ wind speed. In this case, the SCR is no more a drawback for rain measurement. The greater the wind speed, the greater the ocean drawback and thus, the scattering decreases.

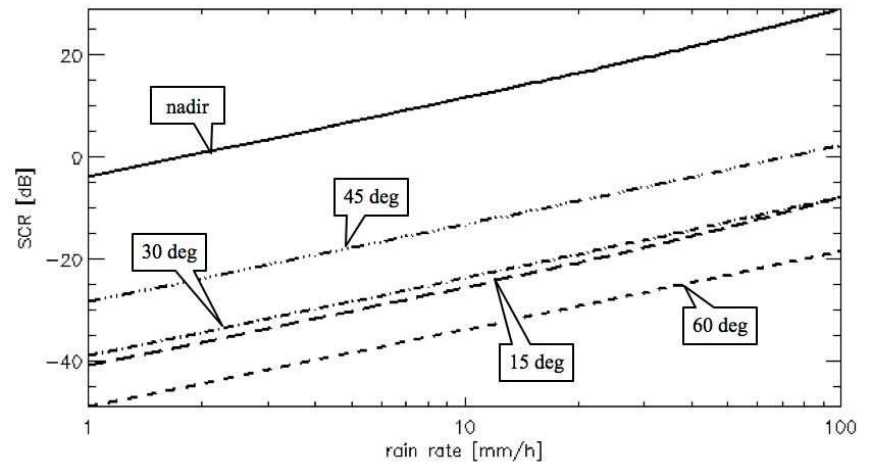

Fig. 7. SCR vs. rain rate over ocean for $24 \mathrm{~m} / \mathrm{s}$ wind speed

\section{CONCLUSION}

TerraSAR-X has good SNR for rain measurement only for nadir-looking geometry. Beyond look angles greater than $15^{\circ}$, the SNR decreases dramatically due to the rain and melting layer attenuation. Nevertheless, in this case, the rain could be estimated indirectly by measuring the bright band peak. The SCR is generally poor except for the nadir region. We conclude that the measurement of rain for nadir-looking geometry is feasible over forest and ocean under almost all conditions. Only for small wind speed and very small rain showers, the estimation of rain over ocean could be problematic. On the other hand, the measurement of rain for side-looking geometry is not feasible except, may be, at very high rain rates. Therefore, the rain measurement experiment should be planned for nadir-looking geometry.

\section{REFERENCES}

[1] R. Meneghini and T. Kozu, Spaceborne weather radar, Artech House, Inc., Boston, 1990.

[2] M. L. Nirala and A. P. Cracknell, The determination of the threedimensional distribution of rain from the Tropical Rainfall Measuring Mission (TRMM) Precipitation Radar, Int. J. Remote Sensing, vol. 23, No. 20, pp. 4263-4304, 2002.

[3] A. R. Jameson, F. Li, S. Durden, Z. S. Haddad, B. Holt, T. Fogarty, E. Im and R. K. Moore, SIR-C/X-SAR Observations of Rain Storms, Remote Sensing of the Environment, vol. 59, pp. 267-279, 1997.

[4] R. K. Moore and A. Ahamad, Limitation on the use of spaceborne imaging SAR as rain radars, Proc. IGARSS, 1993, Tokyo, Japan.

[5] D. Atlas and R. K. Moore, The measurement of precipitation with the synthetic aperture radar, J. of Atmos. and Oceanic Tech., vol. 4, pp. 368-376, 1985

[6] R. E. Rinehart, Radar for meteorologists, Ronald E. Rinehart, Grand Forks, North Dakota, 1991.

[7] F. Fabry and I. Zawadzki, Long-Term Radar Observations of the Melting Layer of Precipitation and Their Interpretation, J. of Atmos. Sciences., vol. 52, No. 7, pp. 838-851, 1994.

[8] A. Bellon, I. Zawadzki, and F. Fabry, Measurements of melting layer attenuation at X-band frequencies, Radio Science, vol. 32, No. 3, pp. 943-955, May-June 1997.

[9] J. C. Curlander and R. C. McDonough, Synthetic Aperture radar: Systems and Signal Processing, John Wiley and Sons, Inc., New York, 1991.

[10] J. Johnson, R. Shin, J. Kong, L. Tsang and K. Pak, A numerical study of the composite surface model for ocean scattering, IEEE Trans. on Geo. and Remote Sensing, vol. 36, pp. 72-83, 1998.

[11] J. W. Pierson and L. Moskowitz, A Proposed spectral form for fully developed wind seas based on similarity theory of $S$. A. Kitaigorodskii, Journal of Geophysical Research, vol. 69, No. 24, pp. 81-90, 1964. 